\title{
Correction to: The chemfp project
}

Andrew Dalke* (D)

\section{Correction to: J Cheminform (2019) 11:76 \\ https://doi.org/10.1186/s13321-019-0398-8}

It was highlighted that the original article [1] contained a mistake in the Abstract and in the legend of Fig. 3. This Correction article shows the incorrect and correct sentence of the Abstract and the correct Fig. 3.

\section{Incorrect sentence}

The same search of 970 million PubChem fingerprints averages $220 \mathrm{~ms}$ /query, making chemfp one of the fastest CPU-based similarity search implementations.

\section{Correct sentence}

The same search of 97 million PubChem fingerprints averages $220 \mathrm{~ms}$ / query, making chemfp one of the fastest CPU-based similarity search implementations.

Published online: 28 September 2020

The original article can be found online at https://doi.org/10.1186/s1332 1-019-0398-8. 


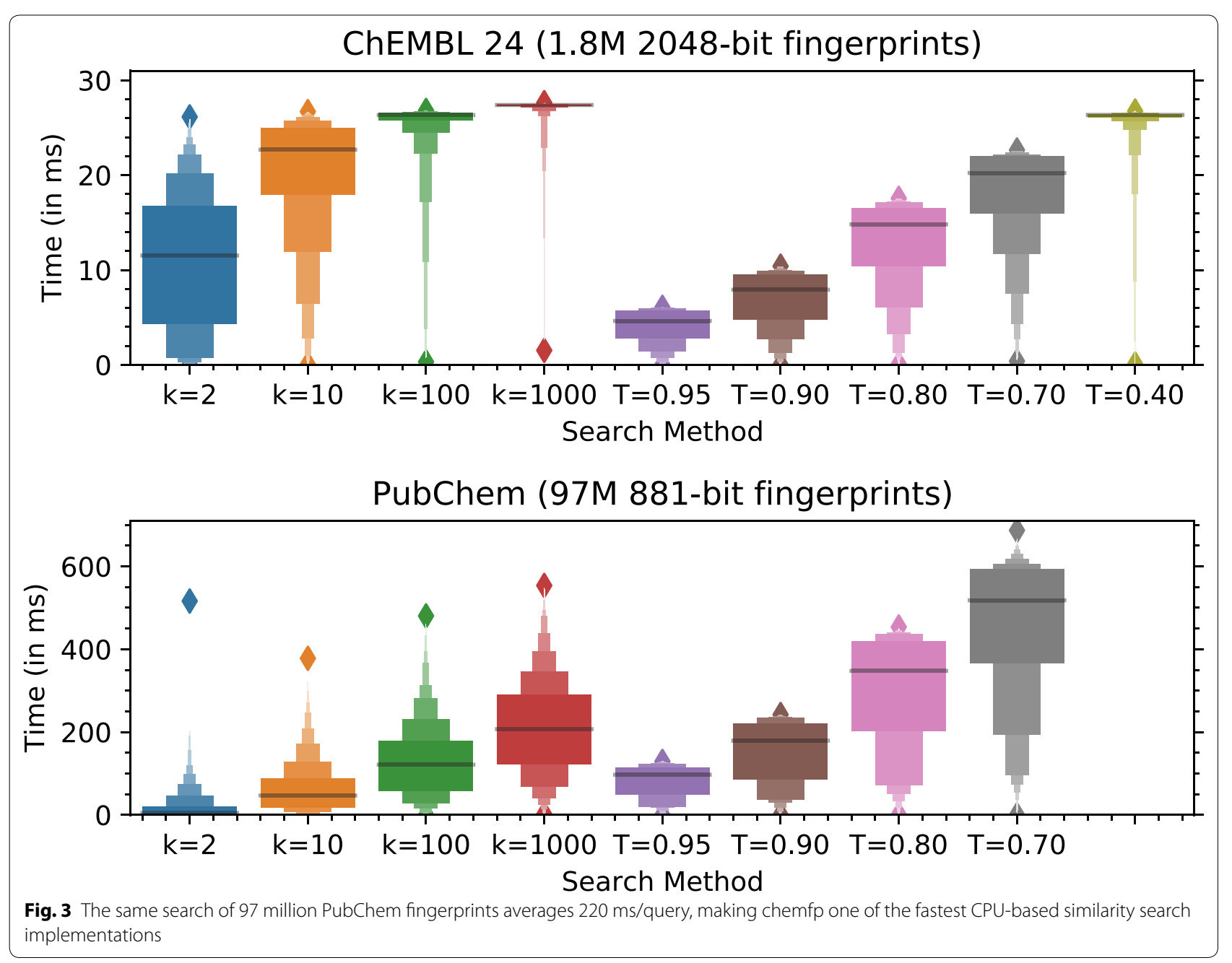

\section{Reference}

1. Dalke A (2019) The chemfp project. J Cheminform. 11:76. https://doi. org/10.1186/s13321-019-0398-8

\section{Publisher's Note}

Springer Nature remains neutral with regard to jurisdictional claims in published maps and institutional affiliations. 